\title{
Spatial Distribution of T-Wave Alternans
}

\author{
D Janusek $^{1}$, M Fereniec $^{1}$, M Kania $^{1}, \mathrm{R}_{\text {Kepski }}{ }^{2}$, R Maniewski ${ }^{1}$ \\ ${ }^{1}$ Institute of Biocybernetics and Biomedical Engineering PAS, Warsaw, Poland \\ ${ }^{2}$ National Institute of Cardiology, Warsaw, Poland
}

\begin{abstract}
The aim of the study was a selection of best ECG leads to get the significant T-wave alternans signal (TWA). The group of 16 patients with implantable cardioverterdefibrillator (ICD) was examined. The 64 lead ECG system was used. Three lead sets were analyzed: 64 BSPM lead set, orthogonal lead set (XYZ) and standard 12 lead set. Patients were divided into two groups: TWA positive (TWA(+)) 5 patients and TWA negative (TWA(-)) 11 patients. In $T W A(+)$ group mean alternans ratio was equal 5.4 (SD 8.09) and in TWA(-) group 0.73 (SD 0.26). Both standard ECG leads and XYZ leads are less sensitive to TWA than specially selected leads from 64 lead set
\end{abstract}

\section{Introduction}

The constant quest for the noninvasive indices which allow risk assessment of ventricular arrhythmia is justified by a large number of sudden cardiac death (SCD) accidents, where ventricular arrhythmias are one of the main reasons [1]. The non-invasive assessment of the cardiac repolarization heterogeneity is of clinical importance. It is generally accepted that the repolarization inhomogeneity facilitates the re-entry phenomena causing the development of life-threatening ventricular arrhythmias, e.g. ventricular tachycardia [2].

The implantable cardioverter-defibrillator (ICD) therapy is of great importance in prevention of SCD nowadays. However, the qualification to ICD therapy is still not an easy task. The noninvasive parameters quantifying depolarization and repolarization process both in time and space are still searched in spite of existing abundance of their kinds.

Among the most useful indices are: low left ventricular ejection fraction, ventricular late potentials, appearance of ventricular arrhythmic episodes in 24-hour Holter electrocardiograms, low heart rate variability, long repolarization duration, and increased QT variability. Recently, the T-wave alterenans (TWA) has been considered as one of the most promising markers, which allows identification patients at an increased risk for ventricular arrhythmia [3]. Repolarization alternans is an electrocardiographic irregularity, which consists in the ventricular repolarization disturbance in consecutive heart beats. TWA is defined as a beat-to-beat change in the T-wave amplitude that repeats every other heart beat. T-wave amplitude variability is usually too small to be observed by visual inspection of the electrocardiogram, what makes a detection of TWA very complicated. Methodology of TWA testing should be improved in order to make an analysis of cycle-to-cycle variability a practical clinical tool for identification those individuals, who are at risk of ventricular fibrillation. Currently, TWA measurements are performed using standard 12 lead ECG or orthogonal XYZ leads. The aim of this study was the selection of best ECG leads from among 64 ECG leads to obtain clinically significant $\mathrm{T}$-wave alternans signal.

\section{Methods}

Electrocardiographic signals were recorded with use of multi-lead high resolution ECG system (Biosemi). Sixty four ECG active electrodes were positioned around the patient's torso according to the University of Amsterdam lead system [4]. Lead location around the torso is shown in figure 1. Measurements were performed in an unshielded room. The ECG signals were digitized with $4096 \mathrm{~Hz}$ sampling frequency and 24 bits amplitude resolution.

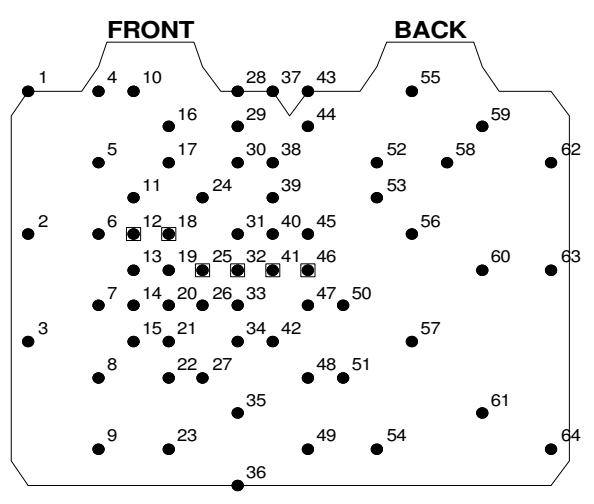

Figure 1. Lead arrangement around the torso. Standard ECG leads are marked by squares. 
The following procedures of signals' processing were applied:

- All disrupted PQRST waves were excluded from the analyzed electrocardiogram (always an even number of beats was removed)

- Baseline wander was eliminated with the use of cubic splines [5]

- QRS complex was detected using the algorithm designed by Pan and Tompkins [6]

- T-waves locations were estimated using Bazett formula [7], with correction on the T-wave maximum location [8]

- FFT-based method was used for estimation of alternans ratio [9]

Sixteen patients with coronary artery disease (CAD) or dilated cardiomyopathy (DCM) were studied (Table 1).

Table 1 . The study group

\begin{tabular}{|c|c|c|}
\hline & TWA(+) & TWA(-) \\
\hline Patients & $5(31.25 \%)$ & $11(68.75 \%)$ \\
\hline Mean age & $57.4(+/-11.74)$ & $61.5(+/-17.49)$ \\
\hline LVEF [\%] & $24.3(+/-6.71)$ & $28.2(+/-12.79)$ \\
\hline CAD & $4(80 \%)$ & $9(81.82 \%)$ \\
\hline DCM & $1(20 \%)$ & $2(18.18 \%)$ \\
\hline
\end{tabular}

All patients had ejection fraction lower than 38\%. Two-minute recordings were made during the ventricular pacing at $100 \mathrm{bpm}$, with use of ICD electrodes. Data set was divided in three subsets according to the lead system used: 64-leads BSPM set, XYZ orthogonal leads set and standard 12 lead ECG set. The results of TWA detection obtained in each subset were compared.

\section{Results}

All patients were divided into two groups: TWA positive $(\mathrm{TWA}(+))$ group consisted of 5 patients and TWA negative (TWA(-)) group consisted of 11 patients. In TWA $(+)$ group, mean alternans ratio was equal 5.4 (SD 8.09) and in TWA(-) group mean alternans ratio was equal 0.73 (SD 0.26). The map of sensitivity of T-wave alternans detection is shown in figure 2 .

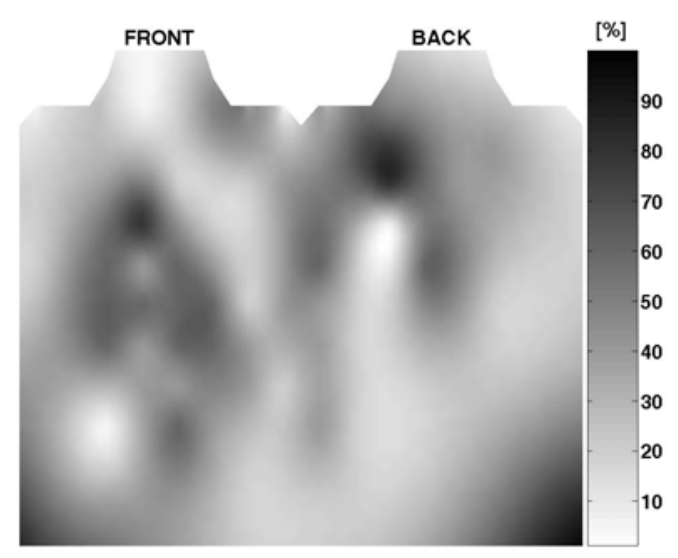

Figure 2. The spatial distribution of detection sensitivity of $\mathrm{T}$-wave alternans.

In the $\mathrm{TWA}(+)$ group alternans ratio exceeded clinically significant value (more than 2.5) in 7 to 57 from among 64 body surface leads. In TWA(-) group, in four patients, alternans ratio exceeded detection threshold in one lead and, in one patient, in two leads. TWA detection sensitivity grater than $80 \%$ was found in the BSPM leads: 11 (lead over V2 lead from standard ECG), 52 (left upper back) and 64 (right lower back). The sensitivity grater than $60 \%$ was found: in $6,7,18,19,20,22,26,45,56$ leads of BSPM system, in X and $\mathrm{Z}$ leads of XYZ lead system and in V2, V3, I, aVF leads of standard ECG lead system. The specificity of TWA detection was equal $100 \%$.

\section{Discussion and conclusions}

Sensitivity of TWA detection strongly depends on the ECG lead location on the body surface. The best criterion for optimal lead selection is the highest TWA signal calculated in single lead. Sensitivity of the TWA detection is strongly correlated with the magnitude of the T-wave or, even more, with the signal to noise ratio. TWA ratio maps shows different patterns of TWA distribution for different patients, but there are leads locations where TWA signal is stronger than in other leads for almost all patients. Preliminary results show that BSPM leads: 11, 52 and 64 are distinctly more sensitive to TWA detection than standard ECG leads. In the case of XYZ lead set the best sensitivity of TWA detection was found in $X$ and $Z$ leads what confirms results of another study, which was done on the group of about 200 patients [10]. For standard 12 lead ECG system, the best sensitivity of TWA detection was found in V2 and V3 leads which are close to BSPM lead number 11.

The results of the study show great advantage of using 
specially selected leads for TWA detection. Due to small number of the recordings obtained results need confirmation by further study on the larger group of patients. There is supposition that T-wave alternans ratio magnitude distribution around the surface of the body might be related to the TWA pattern: concordant or discordant. Indication of another leads location may be helpful for differentiation between patients with one of these two types of TWA.

\section{Acknowledgements}

This work was supported by Ministry of Science and Higher Education, project POL-POSTDOC II $\mathrm{Nr}$ PBZ/MEiN/01/2006/51.

\section{References}

[1] Priori SG, Aliot E, Blomstrom-Lundqvist C, Bossaert L, Breithardt G, Brugada P, and e. al., Update of the guidelines on sudden cardiac death of the European Society of Cardiology, European Heart Journal, vol. 24, pp. 13-15, 2003.

[2] Kuo CS, Munakata K, Reddy CP, Surawicz B, Characteristics and possible mechanism of ventricular arrhythmia dependent on the dispersion of action potential durations, Circulation 1983; 67:1356-67.

[3] Zareba W, Moss AJ, Noninvasive risk strtification in postinfarction patients with severe left ventricular dysfunction and methodology of the MADIT II noninvasive electrophysiology substudy, J Electocardiol. 2003;36 suppl:101-8

[4] Groenwegen S, Spekhorst H, et al. Localization of the Site of Origin of Postinfarction Ventricular Tachycardia by Endocardial Pace Mapping. Body Surface Mapping Compared With the 12-Lead Electrocardiogram. Circulation 1993, 88: 22902306.

[5] Mayer CR, Keiser HN, Electrocardiogram baseline noise estimation and removal using cubic splines and state-space computation techniques, Computers and Biomedical Research 1977, 10, 459-470

[6] Pan J, Tompkins WJ, A real-time QRS detection algorithm, IEEE Trans. Biomed. Eng. 1985, 32, 158-167

[7] Bazett HC, An analysis of the time-relation of electrocardiogram, Heart 1920, 7, 353-370

[8] Karczmarewicz S, Kułakowski P, Janusek D, Buczkowski T, Janusek P, Kociszewska J, Soszczyńska M, Cermużyński L, The new, non-ratedependant algorithm of $\mathrm{T}$-wave identification for time-domain analysis of $\mathrm{T}$ wave alternans, $11^{\text {th }}$
International Congress Cardiostim '98, (Nice, June 17-20, 1998), Proceedings, 174/PW, p.315

[9] Rosenbaum D, Jackson L, Smith J, Garan H, Ruskin J, Cohen R, Electrical alternans and vulnerability to ventricular arrhythmias, N. Engl. J. Med. 1994, 330, $235-241$

[10] Janusek D, Pawłowski Z, Karczmarewicz S, Przybylski A, Comparison of T-wave Alternans Detection Methods, Biocybernetics and Biomedical Engineering, PWN, Warsaw, 2004

Address for correspondence

Dariusz Janusek

Ks. Trojdena 4, 02-109 Warsaw, Poland

djanusek@ibib.waw.pl 